\title{
Anodization behaviour and basic property mapping in the aluminium-erbium system
}

\author{
Andrei Ionut Mardare ${ }^{1,2}$ (D) C Cezarina Cela Mardare ${ }^{1,3}$ - Achim Walter Hassel ${ }^{1,2,3}$
}

Received: 30 June 2017 /Revised: 29 September 2017 / Accepted: 30 September 2017 / Published online: 16 November 2017

(C) The Author(s) 2017. This article is an open access publication

\begin{abstract}
A co-evaporation technique was used for depositing an Al-Er thin film combinatorial library on borosilicate glass substrates with Er concentrations between 3 and 21 at.\% and a total compositional resolution of 0.25 at. $\% \mathrm{~mm}^{-1}$. Scanning droplet cell microscopy was employed for compositional mapping of several fundamental properties of mixed oxides grown on Al-Er thin film alloys. Microstructural and crystallographic particularities of Al-Er alloys were identified along the library, and a relevant compositional threshold at around $\mathrm{Al}-5$ at.\% $\mathrm{Er}$ was identified. Further, small surface grains in the nanometre range were found at $\mathrm{Al}$ grain boundaries, and an initial amorphization and/or hexagonal phase nucleation was identified for Er amounts around 5 at.\%. Electrochemical studies revealed the importance of this compositional threshold, as distinct maximizations of oxide formation factors and oxide electrical permittivities were observed.
\end{abstract}

Keywords Combinatorial libraries $\cdot$ High-throughput experimentation $\cdot$ Scanning droplet cell microscopy $\cdot$ Anodic oxide film $\cdot$ Erbium $\cdot$ Aluminium

Andrei Ionut Mardare

andrei.mardare@jku.at

1 Institute for Chemical Technology of Inorganic Materials, Johannes Kepler University Linz, Altenberger Str. 69, 4040 Linz, Austria

2 Competence Centre for Electrochemical Surface Technology (CEST), Viktor Kaplan Str. 2, 2700 Wiener Neustadt, Austria

3 Christian Doppler Laboratory for Combinatorial Oxide Chemistry (COMBOX) at the Institute for Chemical Technology of Inorganic Materials, Johannes Kepler University Linz, Altenberger Str. 69, 4040 Linz, Austria

\section{Introduction}

Rare earth metals mixed with $\mathrm{Al}$ can form alloys with enhanced mechanical properties, improved extrudability and vibration, heat and corrosion resistance [1,2]. These properties arise from intermetallic phases - formed during bulk alloying processes - that have been found both experimentally and theoretically for many Al-rare earth alloys [3]. In particular, the heavy rare earth element Er induces interesting property changes in alloys with $\mathrm{Al}$ resulting from synergetic effects on precipitates that affect resistance to recrystallization and the as-induced hardening of the alloys $[4,5]$. Further, grain refinement has recently been observed in bulk Al-Er alloys during extrusion forming, as precipitates are generated that limit grain boundary migration. This can be used to increase resistance to electromigration in $\mathrm{Al}$ alloys [6, 7]. Additionally, Er-induced modifications of technologically relevant $\mathrm{Al}$ alloys (e.g. containing $\mathrm{Mg}$ ) to improve their mechanical and corrosion behaviour via microstructural tuning have been demonstrated [8-11].

Er is also an interesting alloying element for tuning properties of oxides: glass formation and polyamorphism have already been reported [12-15]. While the influence of $\mathrm{Al}$ on $\mathrm{ZnO}$ for further improvement of transparent conductive oxides is well studied, addition of $\mathrm{Er}$ has been demonstrated to allow tuning of $\mathrm{ZnO}$ electrical properties [16]. Further, (Al, Er) codoped $\mathrm{ZnO}$ nanoparticles have recently been used to achieve high rates of photocatalytic degradation of various molecules (e.g. methyl orange, rhodamine blue), with direct applications in optoelectronic devices $[17,18]$. Photoluminescence induced by $\mathrm{Er}^{3+}$ after doping of $\mathrm{Al}_{2} \mathrm{O}_{3}$ has been demonstrated, and the influence of various mixed $\mathrm{Al}-\mathrm{Er}$ oxides on the emission in relation to the Er amount has been discussed [19]. The use of Er-doped $\mathrm{Al}_{2} \mathrm{O}_{3}$ in the fabrication of optical waveguides via various techniques has been reported, including atomic layer 
deposition, reactive co-sputtering and chemical vapour deposition [20-22].

The chances of finding new applications in the Al-Er system - for both metallic and mixed oxide states - are therefore high. Previous studies of alloys from this system lack a systematic investigation of particular properties of interest in the context of an Al-Er compositional spread. However, compositional mapping of combinatorial libraries is becoming increasingly popular, as these libraries are useful in identifying compositions of interest for various applications, for instance, as materials in medicine, electronics, water splitting and photovoltaics [23-26]. Against this background, we performed a compositional mapping focussing on the basic properties of Al-Er alloys and their anodic oxides. A similar study involving rare earths - more specifically, the Al- $\mathrm{Tb}$ system - has recently identified various properties that bulk alloy studies were unable to predict [27].

\section{Experimental details}

The Al-Er metallic alloys were deposited on borosilicate glass substrates $\left(26 \times 76 \mathrm{~mm}^{2}\right.$, VWR International $\left.\mathrm{GmbH}\right)$ using a thermal co-evaporator developed in house. The vacuum chamber was part of a larger vacuum cluster system and included a load lock, other preparation chambers and various analytical units. Prior to each deposition, the substrates were cleaned sequentially in acetone, isopropanol and water by ultrasonication. Two thermal evaporation sources containing individual high-purity Al (99.95\% Goodfellow) and Er (99.9\% Sigma-Aldrich) metals were simultaneously electrically powered using high-current DC power supplies. The subsequent vapour-phase mixing of both $\mathrm{Al}$ and Er species combined with off-axis positioning of each source led to the formation of an Al-Er compositional gradient along the substrates. The base pressure of the vacuum system was in the range of $10^{-5} \mathrm{~Pa}$, and during deposition, pressure was increased to up to $10^{-3} \mathrm{~Pa}$. For $\mathrm{Al}$ evaporation, a $\mathrm{W}$ boat was used as heating element, while a small BN crucible (approx. $1 \mathrm{~cm}^{3}$ ) heated by a helicoidal W filament was employed in Er evaporation. Both sources were positioned at a distance of $120 \mathrm{~mm}$ from the substrates, and the thermal load due to radiation during deposition resulted in a substrate temperature increase up to $335 \mathrm{~K}$. Separate crystal quartz microbalances (QCMs, Inficon) positioned above each metallic source allowed the individual $\mathrm{Al}$ and Er deposition rates to be controlled in situ. These rates were kept constant for the entire deposition time at 1 and $0.2 \mathrm{~nm} \mathrm{~s}^{-1}$, respectively, since a low amount of Er was desired to co-deposit with Al. A total cumulative Al-Er thin film thickness of $500 \mathrm{~nm}$ was obtained after 7 min of co-deposition, as indicated by both QCMs. A maximum thickness deviation of $10 \%$ can be expected along the compositional spread due to individual $\mathrm{Al}$ and Er atomic spatial distributions during vapour-phase mixing.

Immediately after Al-Er co-deposition, the library was transferred without breaking vacuum into a customized scanning energy-dispersive X-ray (SEDX) spectroscopy system attached to the vacuum cluster chambers. An in-vacuum Gantry robot designed in house scanned the sample under irradiation from an electron beam (emitted by a JSM-T330 JEOL electron column) along the surface. A Si drift detector (SDD, remX $\mathrm{GmbH}$ ) was used for X-ray detection. At an acceleration voltage of $20 \mathrm{kV}$, electrons were de-focalized uniformly to irradiate a circular spot of $500 \mu \mathrm{m}$ in diameter. This procedure minimized the substrate influence on the EDX quantitative analysis by dramatically increasing the signal-tonoise ratio for $\mathrm{Al}$ and Er. Programming of the scanning procedures, calibration and data acquisition were done in house using LabView. The quantitative elemental analysis performed at various locations along the entire library (using IDFix software from remX GmbH implemented in LabView) allowed simultaneous mapping of $\mathrm{Al}$ and $\mathrm{Er}$ amounts obtained along the compositional gradient. This information was further used to locate individual Al-Er alloys on the surface of the library. The Er amount measured along the Al-Er thin film compositional spread varied between 3 and 21 at.\%. This corresponded to a compositional resolution of approximately 0.25 at. $\% \mathrm{~mm}^{-1}$, which was ideal for further analysis of individual Al-Er alloys, as the lateral spacing amounted to $4 \mathrm{~mm}$ for each at.\% of composition change.

Microstructure and crystallographic particularities of individual Al-Er alloys were mapped along the compositional gradient using scanning electron microscopy (SEM) and scanning X-ray diffraction (SXRD), respectively. The surfaces of various alloys were imaged using a field emission Zeiss Gemini 1540 XB SEM with $20 \mathrm{kV}$ acceleration voltage and in-lens detection. A PANalytical X'pert Pro XRD system was operated in grazing incidence $\left(0.4^{\circ}\right.$ angle, $\mathrm{Cu}-\mathrm{K}_{\alpha}$ source $)$ mode to scan the library with the compositional gradient.

Electrochemical studies were performed along the Al-Er library using a scanning droplet cell microscope (SDCM). Details of the 3D printing of the full three-electrode cell configuration and of the SDCM flow channels can be found elsewhere [28]. The SDCM was attached to a Gantry robot designed in house, which automatically scanned the surface of the library using LabView software. As counter electrode, a $\mathrm{Au}$ wire was wound around the glass capillary holding a $\mu$ $\mathrm{AuHg} / \mathrm{Hg}_{2}\left(\mathrm{CH}_{3} \mathrm{COO}\right)_{2} / \mathrm{NaCH}_{3} \mathrm{COO}$ reference electrode $(\mu-$ $\mathrm{RE}$ ), and together, these were inserted into the SDCM body. Full details about the fabrication of the $\mu$-RE have previously been reported [29]. The potential of the $\mu-\mathrm{RE}$ was $0.505 \mathrm{~V}$ vs. the standard hydrogen electrode (SHE), and all potentials used and/or discussed in this work are given with reference to SHE. To ensure reproducibility of the wetted area while analysing various Al-Er alloys, the SDCM was used in contact mode. To 
this end, the electrolyte was confined inside the tip of the cell by using a soft sealing combined with a reproducible downward force $(4 \mathrm{~N})$ measured by a force sensor working in a PID control loop with the Z Gantry motor. Before experimentation on Al-Er thin film alloys, an area of $3.821 \mathrm{~mm}^{2}$ (corresponding to a diameter of approximately $2.2 \mathrm{~mm}$ ) was optically measured using the same SDCM to analyse a coloured oxide spot grown on a Ti thin film [30].

All electrochemical experiments were performed using borate electrolyte buffered at a $\mathrm{pH}$ value of 8.0, which was obtained by mixing analytical-grade aqueous solutions of $\mathrm{H}_{3} \mathrm{BO}_{3}$ and $\mathrm{NaOH}$. The SDCM was connected to a CompactStat potentiostat (Ivium Technology) electrochemical system featuring an integrated frequency response analyser. Individual alloys along the Al-Er library were anodised potentiodynamically at a scan rate of $100 \mathrm{mV} \mathrm{s}^{-1}$. The maximum potential applied in each cyclic voltammogram (CV) was increased in $1 \mathrm{~V}$ steps up to $10 \mathrm{~V}$ vs. SHE. Before each step of anodic oxide thickness increase, electrochemical impedance spectroscopy (EIS) was performed at $0 \mathrm{~V}$ in a frequency window ranging from $10^{5}$ to $10^{-1} \mathrm{~Hz}$ using a superimposed AC perturbation of $50 \mathrm{mV}$.

\section{Results and discussion}

The main goal of this study was to investigate the electrochemical behaviour of Al-Er thin film alloys. However, anodization requires a thorough understanding of the specific properties of the parent metal alloys. The crystallographic particularities of Al-Er thin film alloys were investigated by XRD along the compositional spread; Fig. 1 shows selected diffractograms as a function of alloy composition. The Er content of the selected alloys is indicated on the vertical axis on the right side of the figure. Additionally, the diffractograms of pure $\mathrm{Al}$ and pure Er thin films (deposited under similar conditions as the Al-Er library) are presented as references.

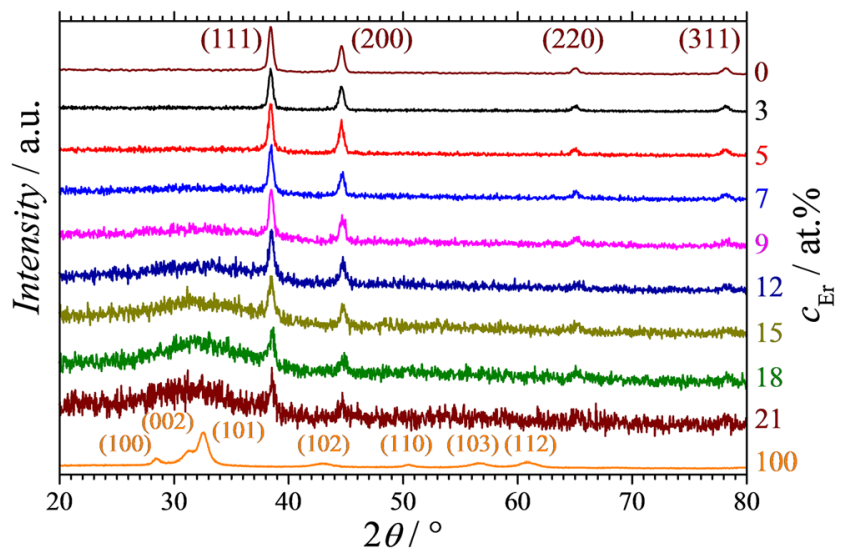

Fig. 1 Selected X-ray diffractograms corresponding to various Al-Er alloys and pure $\mathrm{Al}$ and $\mathrm{Er}$ as reference
Pure Er exhibits hexagonal symmetry, with seven distinct peaks indexed in accordance with the powder diffraction file (PDF 00-002-0930) database. The well-known cubic structure of Al can be clearly observed for both the pure film and for alloys with low Er contents (e.g. 3 at.\%). The high signal-tonoise ratio indicates that low Er amounts do not significantly distort the Al lattice. However, increasing Er concentration along the library resulted in a decrease in the signal-to-noise ratio, as can be seen when comparing, for instance, the diffractograms corresponding to 21 and 3 at. $\%$ Er.

A compositionally induced gradual decrease in all cubic peaks is clearly observable, and the (220) and (311) peaks disappear almost completely at concentrations above approximately 15 at.\% Er. Any decrease in the XRD peak intensity can usually be related to a decrease in crystallinity (and/or a change of a certain phase), which is in this case supported by the appearance of a very broad peak centred approximately at a $2 \theta$ value of $32^{\circ}$. Such peaks are always an indication of incipient thin film amorphization, especially when substrate contribution is minimized by using grazing incidence XRD. This broad peak is distinctly visible in the Al-Er library at Er concentrations above 9 at.\%. Indications of broad peak formation can also be found at 7 at.\% Er in background-referenced deconvolution (not shown here). Even though the alloys are not completely amorphous, mixing cubic with hexagonal materials results in a strong distortion of the crystalline lattice. Such effects have previously been observed in other binary and ternary thin film libraries when mixing dissimilar symmetries at the atomic level in the vapour phase [25, 27].

Along the entire Al-Er combinatorial library, no evidence of intermetallic phase formation was found, which supports the idea of an intimate atomic mixing of $\mathrm{Al}$ and Er during film growth that may not necessarily be reproducible in bulk alloys. Further, the evolution of Al peaks with increasing Er amount indicates the occurrence of phase mixing (based on cubic $\mathrm{Al}$ ). The possibility of phase segregation can be safely excluded based on the previously mentioned atomic mixing in the vapour phase combined with the low kinetic energy of the evaporand atoms (due to thermal evaporation) and their condensation at room (i.e. low) temperature. The origin of the second phase that mixes with the cubic one is not clear. The broad XRD peak centred at $32^{\circ}$ may indicate an initial step of hexagonal phase nucleation, since the most intense hexagonal Er peaks are observed at the same position. This peak is also characteristic of an amorphous phase, as previously discussed. In either case, it can be concluded that a distortion of the $\mathrm{Al}$ cubic symmetry propagates throughout the entire Al-Er alloy range analysed here. This idea is also supported by a slight shift of all XRD peaks with increasing Er amount, as closer analysis of Fig. 1 shows.

The microstructure analysis of the Al-Er thin film combinatorial library revealed that the surface changes slightly with changing composition. Figure 2 presents a collection of 


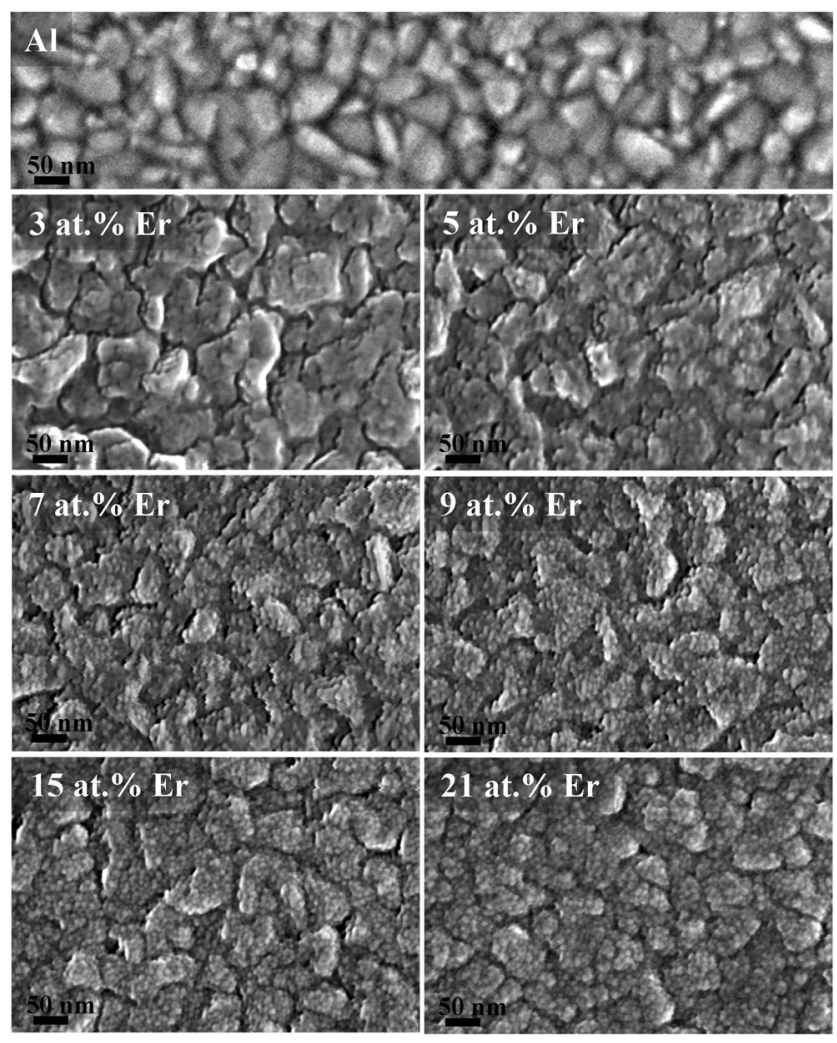

Fig. 2 SEM imaging of Al-Er alloy microstructure for different Er contents and pure $\mathrm{Al}$ surface

selected SEM images taken at various locations along the compositional gradient. For very low Er amounts, the surface microstructure closely resembles the pure Al surface presented at the top of Fig. 2. Typical grains in the range of $100 \mathrm{~nm}$ can be observed for all alloys. Additionally, the presence of much finer grains decorating the larger ones can be observed along the compositional gradient. These finer grains start to emerge at 7 at.\% Er and are uniformly distributed across the examined surface. A new type of grain structure can typically be expected to nucleate first at the energetically most favourable places (i.e. grain boundaries). The SEM images corresponding to 5 at.\% Er tend to support this, as do the conclusions of previous studies, which indicate that Er acts to mediate grain refinement in $\mathrm{Al}$ alloys by limiting grain boundary migration [6]. The surface density of the finer grains does not appear to increase much with increasing Er concentrations. All alloys above 5 at.\% Er have a very similar microstructure. Even though the volume density of the small grains cannot be determined from Fig. 2, a closer investigation of the SEM images (comparing, e.g. the surface of 5 or 7 at.\% $\mathrm{Er}$ with that at 21 at.\% Er) suggests that higher levels of grain boundary filling occur with increasing Er amounts.

The compositional region around 5 at.\% Er corresponds to possible nucleation of the hexagonal phase and/or beginning amorphization, as suggested by analysis of the XRD data shown Fig. 1. However, although the microstructural property mapping matches the crystallography of the Al-Er alloys well, the amorphization and/or hexagonal phase nucleation question cannot be answered conclusively. This is because, on the one hand, the smaller grains decorating larger ones may constitute a new (i.e. hexagonal) crystalline phase, while, on the other hand, any amorphization process would result directly in the formation of much finer grains on the surface (or their complete absence). However, that the size of the decorating grains does not increase with increasing Er content despite the broad XRD peak clearly increasing (see Fig. 1) may indicate a mixture of the two phases. The absence of well-defined hexagonal peaks suggests with a higher probability that the second phase is amorphous. This matches well the conclusions from the analysis of the XRD mapping alone and can be considered a trend in the Al-Er alloy microstructure. The microstructural and crystallographic changes identified at 5 at. $\% \mathrm{Er}$ indicate the presence of a relevant compositional threshold arising from increasing amounts of the amorphous phase during its mixing with the cubic one. Distinct peaks or inflexion points in property mappings usually reflect compositional thresholds where $3 \mathrm{D}$ atomic networks of a minor new phase form in an $\mathrm{Al}$ alloy [31, 32].

Using SDCM, the electrochemical behaviour of the Al-Er thin film combinatorial library under study was mapped along the compositional gradient. The choice of 8.0 as the electrolyte $\mathrm{pH}$ value was a compromise between the passive regions of $\mathrm{Al}$ and Er. This is revealed by the analysis of the corresponding Pourbaix diagrams [33]. First, the open circuit potential (OCP) was mapped along the library as an indirect measure of possible surface energy and/or corrosion potential changes induced by the Er amount in $\mathrm{Al}$ [34]. This was done by positioning the SDCM tip at several locations along the library. The measured OCP was weakly dependent on the composition (variations in the $\mathrm{mV}$ range), and an average potential value of $524 \mathrm{mV}$ vs. SHE was measured for all alloys (not shown here).

The Al-Er compositional spread was anodised potentiodynamically in a stepwise manner at up to $10 \mathrm{~V}$, and the results were mapped as a function of composition. In Fig. 3, several series of CVs measured for different Al-Er alloys are presented. For all measurements, the valve metal behaviour of the alloys (characteristic of $\mathrm{Al}$ ) can be observed as a sudden current rectification at the end of each $\mathrm{CV}$, where the potential begins to decrease. Thus, the anodic oxides grow following the high-field model, and the constant rate of potential increase implies a constant oxidation current plateau [35]. Further, given the identical stoichiometry of the oxidized forms of $\mathrm{Al}$ and $\mathrm{Er}$ (both having stable 3+ states), a mixed oxide formation would be expected during anodization. The oxidation current plateau is not directly observable in Fig. 3, but is hinted at by the highest current density values, which are almost constant for each $\mathrm{CV}$ series of a specific alloy.

A certain dynamic can be followed along the compositional gradient that points towards compositionally dependent Al-Er 


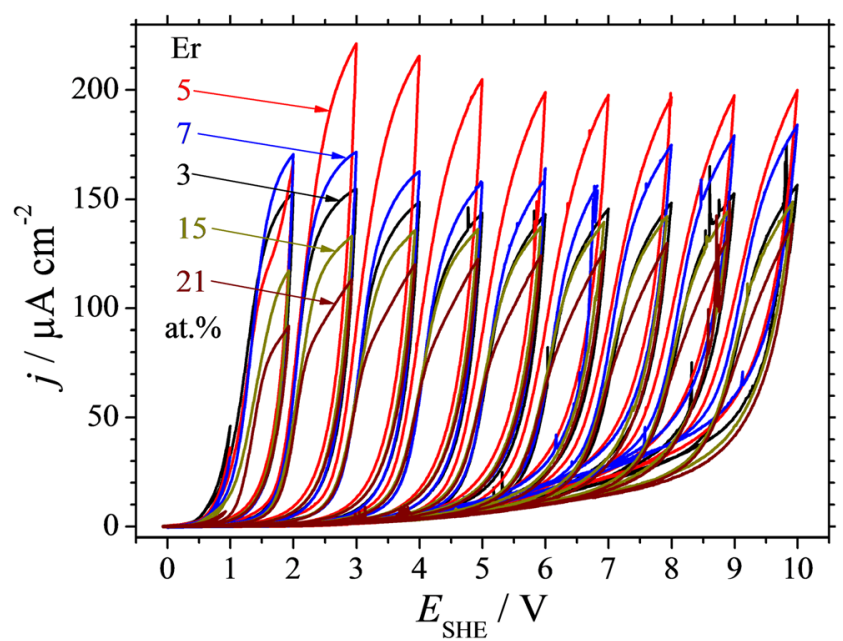

Fig. 3 Series of CVs performed during potentiodynamic oxide growth $\left(100 \mathrm{mV} \mathrm{s}^{-1}\right)$ at various Er concentrations in the Al-Er system

anodization particularities. The CV series corresponding to Al-5 at.\% Er shows the highest current densities, which supports the idea of a distinct compositional (percolation) threshold for this Er amount. Below and above this threshold, the current density values are lower and change gradually with the Er content. This suggests that the phase mixing of the parent metal alloys directly affects the mixing of the anodic oxides during anodization. Higher current density plateaus at the compositional threshold (Al-5 at.\% Er) indicate that thicker mixed oxide grows per each volt applied. Attributing this effect to a single cause may be difficult. In fact, as soon as a minor phase stabilizes in a mixture with a major phase, many variables change simultaneously, such as lattice parameters, binding energies, density, overall electrical properties and surface energy, each of which may directly influence oxide growth. Moreover, synergetic effects are always to be expected when crossing such compositional thresholds, as shown previously in numerous electrochemical combinatorial studies [23-28, 30-32].

A deeper analysis of the CV series allows the oxide formation factors to be calculated as material constants depending on the parent metallic alloy composition. To this end, the CVs in the time domain (not shown here) were integrated, and the charge consumed during oxide growth was evaluated. A high current efficiency was assumed as specific to $\mathrm{Al}$ [35]. The amount of charge corresponding to each step of oxide thickness increase was additively calculated in order to extract an initial charge oxide formation factor. The corresponding plots are presented in Fig. 4, where the charge densities are given in relation to the anodization potential for selected Al-Er alloys. From the slope of each curve, a charge oxide formation factor can be extracted that describes (in $\mathrm{mC} \mathrm{cm}^{-2} \mathrm{~V}^{-1}$ ) the charge necessary for growing oxide on a specific Al-Er alloy. However, a deviation from linearity can be observed in Fig. 4. Such deviations are sometimes encountered in coulometry and may be due to

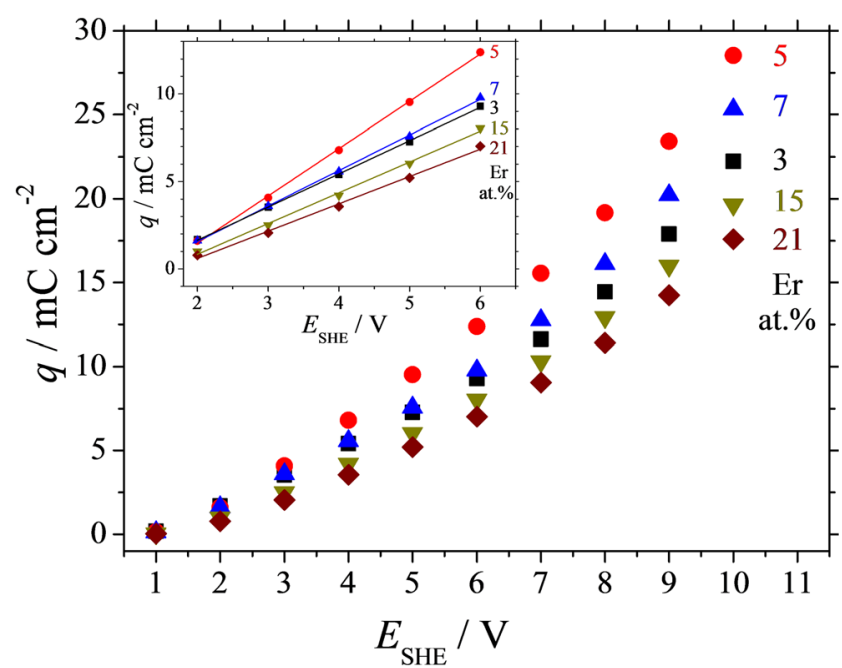

Fig. 4 Charge density consumed during anodic oxide formation on AlEr alloys and (inset) potential region used for linear regression of the experimental data

multiple factors that depend on composition, such as current efficiency, surface wettability, gas evolution and stability/dissolution. The inset in Fig. 4 plots selected experimental data points for anodization potentials up to $6 \mathrm{~V}$. In this range, good linearity is observed, and the slopes of the curves can therefore be used to map the anodic oxide formation factors along the AlEr thin film combinatorial library.

Using Faraday's law (describing the direct proportionality of charge density to mass) allows the charge consumed during oxide formation to be converted into the mass of the mixed anodic oxide. Further, oxide thickness can be calculated given the area investigated by SDCM and using compositionally dependent values of oxide densities. Mixed matter theory was applied to estimate individual oxide densities based on the atomic ratios of $\mathrm{Al}$ and $\mathrm{Er}$ parent alloys, using values of 3.50 and $8.64 \mathrm{~g} \mathrm{~cm}^{-3}$ for $\mathrm{Al}_{2} \mathrm{O}_{3}$ and $\mathrm{Er}_{2} \mathrm{O}_{3}$, respectively [27]. On the basis of this approach, the oxide formation factor can be obtained (in $\mathrm{nm} \mathrm{V}^{-1}$ ); its compositional mapping along the Al-Er library is presented in Fig. 5. As in previous situations, the special compositional threshold is evidenced by radical changes. A distinctive peak can be observed around 5 at.\% Er. The formation factors are relatively constant along the entire compositional spread except in this region, which describes a microstructural transition, as discussed above.

That the oxide formation factors are below the known value of pure $\mathrm{Al}_{2} \mathrm{O}_{3}\left(1.6 \mathrm{~nm} \mathrm{~V}^{-1}\right)$ may be due to the assumption of maximum current efficiencies. As previously mentioned, the choice of electrolyte $\mathrm{pH}$ was a compromise balancing the passive regions of both $\mathrm{Al}$ and Er. However, additional processes that occur simultaneously with anodic oxide formation (e.g. gas evolution, $\mathrm{Al}$ and/or Er dissolution) cannot be excluded completely. This is clearly observable in the deviation from linear behaviour in the curves of Fig. 4. Further, since a material's particular behaviour/property during 


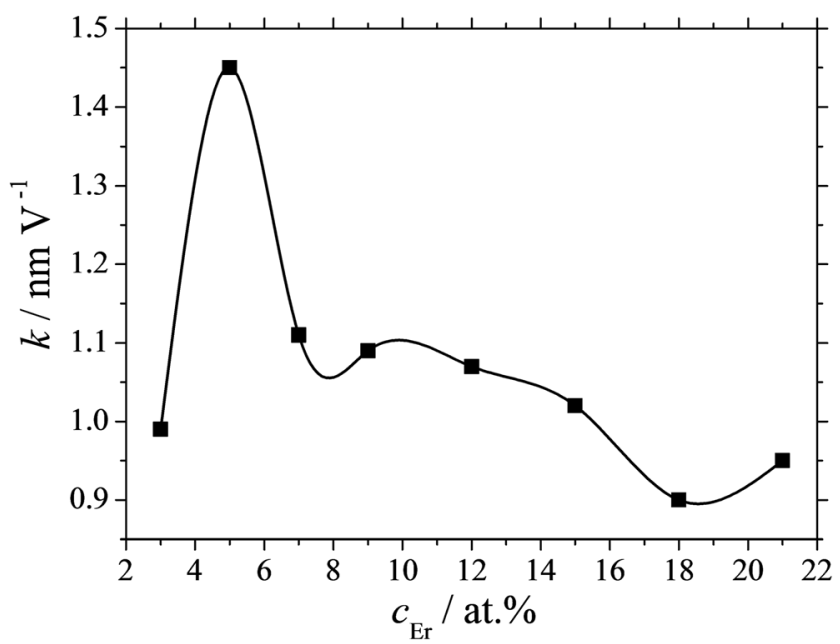

Fig. 5 Compositional mapping of the oxide formation factors on Al-Er thin film alloys

compositional mapping is identified by direct comparison with the neighbouring alloys, composition-induced evolution of this property is more relevant than its absolute scale.

In order to provide a full electrochemical characterization of Al-Er alloys, EIS was performed before each anodization step. This provides information about the electrical behaviour of mixed oxides grown on individual alloys. Series of EIS datasets were measured by SDCM at each Al-Er alloy spot investigated along the compositional spread. An example of a Bode representation is given in Fig. 6 for the anodic oxide grown on Al-7 at.\% Er. The arrows indicate the impedance and phase evolution with increasing anodization potential. In the low-frequency range, a clear increase in impedance due to an increase in oxide thickness is observed at higher potentials. For each Al-Er alloy analysed, a single time constant was always present and a typical equivalent circuit containing an electrolyte resistance $R_{\mathrm{el}}$ in series with a parallel $R_{\mathrm{ox}} C_{\mathrm{ox}}$ couple describing the oxide was identified. However, the phase shifts reached only a minimum of $-85^{\circ}$, which indicates that a

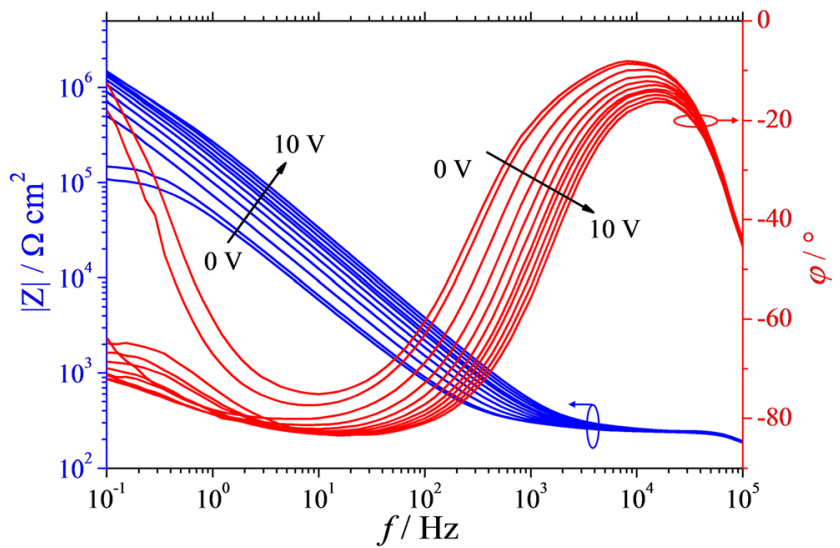

Fig. 6 Representative Bode plot of the EIS performed after each anodization step during oxide formation on Al-Er alloys constant phase element (CPE) should be preferred over an ideal capacitance $C_{\mathrm{ox}}$. The approximately -1 slope of the curves (independent of the applied potential) directly confirms the correct use of such an equivalent circuit.

The EIS data alone is of limited use in compositional mapping, but upon fitting each of the curves, the true oxide capacitance $C_{\mathrm{ox}}$ can be calculated. Such a fitting procedure was performed for all EIS data measured. The exponents of the CPEs had values ranging from 0.85 to 0.95 . The true oxide capacitance (preferably independent of frequency) can be calculated following the $\omega_{\max }$ route [36] or the Cole-Cole route involving the $R_{\mathrm{ox}}$ value [37]. The former approach was ruled out, since - due to the particularities of the system under study - in Nyquist representation (not shown here), $\omega_{\max }$ cannot be identified. The latter approach was applied, and the capacitance results obtained were very close to those directly described by the value of the CPE (due to high exponent values close to 1). Additionally, the capacitances corresponding to the frequency of maximum phase shift observable at $10 \mathrm{~Hz}$ in Fig. 6 were calculated. These values were lower than both the CPE and the Cole-Cole values. Since the capacitance values directly expressed by the CPE came close to averaging the results obtained via the other routes, they were used further as the capacitances of Al-Er mixed oxides.

A plot of the inverse capacitance as a function of the anodization potential allows calculation of the oxide dielectric constant, which depends on the manner in which $\mathrm{Al}_{2} \mathrm{O}_{3}$ and $\mathrm{Er}_{2} \mathrm{O}_{3}$ mix during oxide formation on a particular Al-Er alloy. Figure 7 shows several selected inverse capacitance plots in 3D representation. The linear behaviour of each curve can clearly be seen. The definition of capacitance was used for direct calculation of the mixed oxide dielectric constants as functions of Al-Er composition. Using the high-field formulation of the relation between oxide thickness and anodization potential allowed direct calculation of the natural oxide thickness for each Al-Er alloy analysed [35]. Since the slopes of the

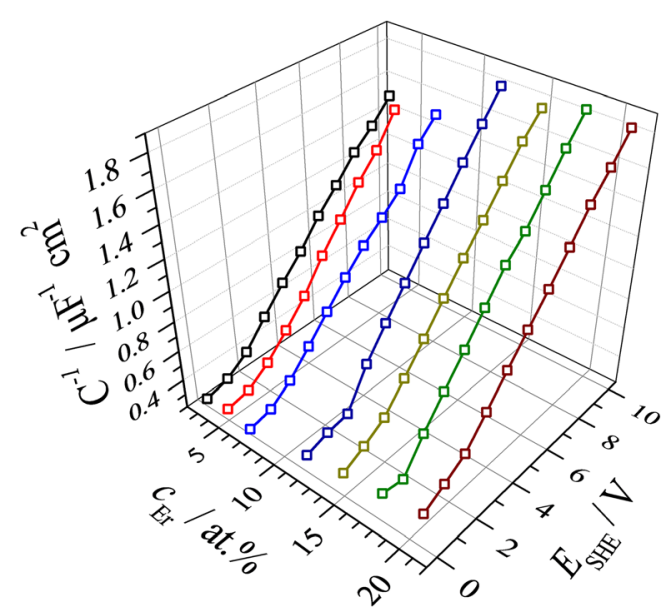

Fig. 7 Relation between anodic oxide inverse capacitance and anodization potential for selected Al-Er alloys 


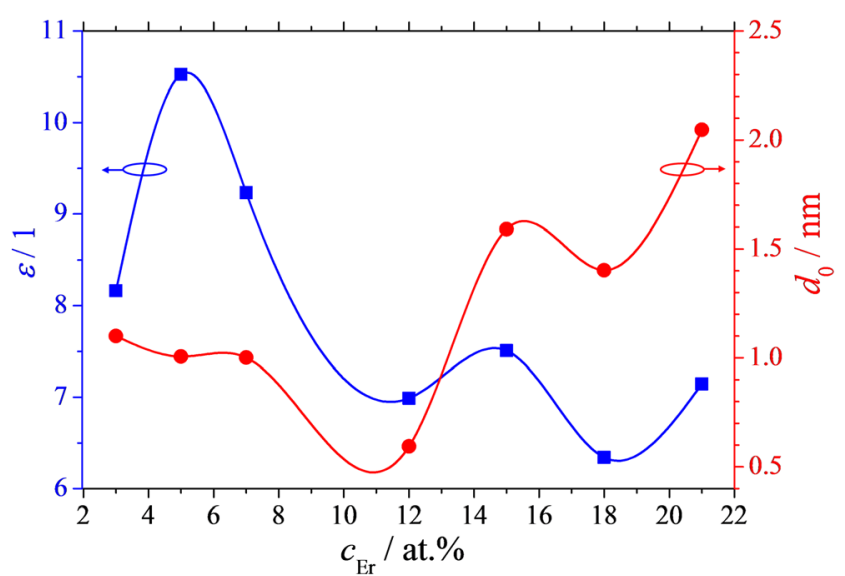

Fig. 8 Compositional mapping of anodic oxide electric permittivity and natural oxide thickness for the Al-Er system

inverse capacitance curves allow the permittivities to be calculated, their intercepts provide access to the natural oxide thicknesses. Corresponding property mappings are presented in Fig. 8 for the entire range of Al-Er alloys under study. Both the dielectric constant $\varepsilon$ (left vertical scale) and natural oxide thickness $d_{0}$ (right vertical scale) are compositionally mapped. With increasing Er amount in the library, the anodic oxides show an increase in the dielectric constant, with a peak at 5 at.\% Er. Again, the transitional composition is distinctly visible, but this time in the electrical behaviour of the mixed Al-Er oxides. Following this enhancement, with increasing Er content, the dielectric constant drops to values around 7 and remains relatively independent of composition. The calculated natural oxide thickness does not follow the same trend. Values ranging between 0.5 and $2.0 \mathrm{~nm}$ were obtained throughout the library, but no distinct peak was observed at the transitional composition. This may suggest that the main species contributing to the natural oxides on Al-Er alloys is Al, since the strong transitional influence of Er was not observed here.

\section{Conclusions}

Several fundamental properties of oxides grown on Al-Er thin film alloys were compositionally mapped using scanning droplet cell microscopy. For this purpose, an Al-Er thin film combinatorial library was deposited on glass substrates using a co-evaporation technique. The microstructural and crystallographic particularities of the Al-Er parent metal alloys indicated the presence of a distinct compositional threshold at Al5 at.\% Er. Nanometre-sized surface grains nucleating at the original $\mathrm{Al}$ grain boundaries together with an incipient amorphization and/or hexagonal phase nucleation were identified at this composition. The importance of the identified compositional threshold was evident in electrochemical anodization studies of the Al-Er alloys, where distinct maximizations of oxide formation factors and oxide electrical permittivities were observed.

Acknowledgements Open access funding provided by Johannes Kepler University Linz. The authors thank Isabella Pötzelberger and Carina Grill for their assistance in experiments in the initial phase of the study.

Funding information The financial support by the Austrian Federal Ministry of Science, Research and Economy and the National Foundation for Research, Technology and Development through funding of the Christian Doppler Laboratory for Combinatorial Oxide Chemistry (COMBOX) is gratefully acknowledged.

Financial support from the Competence Centre for Electrochemical Surface Technology GmbH (CEST) within the scope of the strategic project ELMIGAL is gratefully acknowledged.

Open Access This article is distributed under the terms of the Creative Commons Attribution 4.0 International License (http:// creativecommons.org/licenses/by/4.0/), which permits use, duplication, adaptation, distribution and reproduction in any medium or format, as long as you give appropriate credit to the original author(s) and the source, provide a link to the Creative Commons license, and indicate if changes were made.

\section{References}

1. Ping D, Hono K, Inoue A (2000) Microstructural characterization of a rapidly solidified ultrahigh strength $\mathrm{Al}_{94.5} \mathrm{Cr}_{3} \mathrm{Co}_{1.5} \mathrm{Ce}_{1}$ alloy. Metall Mater Trans A 31:607-614

2. Lundin R, Wilson JR (2000) Rare earth metals. Adv Mater Process 158:52-55

3. Jin L, Kanga Y-B, Chartrand P, Fuerst CD (2010) Thermodynamic evaluation and optimization of Al-Gd, Al-Tb, Al-Dy, Al-Ho and Al-Er systems using a modified quasichemical model for the liquid. Calphad 34:456-466

4. Li H, Gao Z, Yin H, Jiang H, Su X, Bin J (2013) Effects of Er and Zr additions on precipitation and recrystallization of pure aluminum. Scripta Mater 68:59-62

5. Wen SP, Gao KY, Li Y, Huang H, Nie ZR (2011) Synergetic effect of $\mathrm{Er}$ and $\mathrm{Zr}$ on the precipitation hardening of Al-Er-Zr alloy. Scripta Mater 65:592-595

6. Su N, Guan R, Wang X, Wang Y, Jiang W, Liu H (2016) Grain refinement in an Al-Er alloy during accumulative continuous extrusion forming. J Alloys Comp 680:283-290

7. Nie Z, Jin T, Fu J, Xu G, Yang J, Zhou J, Zuo T (2002) Research on rare earth in aluminum. Mater Sci Forum 396-402:1731-1736

8. Seetharaman S, Blawert C, Milton Ng B, Wong WLE, Goh CS, Hort N, Gupta M (2015) Effect of erbium modification on the microstructure, mechanical and corrosion characteristics of binary Mg-Al alloys. J Alloys Comp 648:759-770

9. Rosalbino F, Angelini E, De Negri S, Saccone A, Delfino S (2005) Effect of erbium addition on the corrosion behaviour of Mg-Al alloys. Intermetallics 13:55-60

10. Rosalbino F, Angelini E, De Negri S, Saccone A, Delfino S (2003) Influence of the rare earth content on the electrochemical behaviour of Al-Mg-Er alloys. Intermetallics 11:435-441

11. Rosalbino F, Angelini E, De Negri S, Saccone A, Delfino S (2006) Electrochemical behaviour assessment of novel Mg-rich Mg-Al-RE alloys $(\mathrm{RE}=\mathrm{Ce}, \mathrm{Er})$. Intermetallics 14:1487-1492

12. Weber JKR, Abadie JG, Hixson AD, Nordine PC, Jerman GA (2000) Glass formation and polyamorphism in rare-earth oxide-aluminum oxide compositions. J Am Ceram Soc 83:1868-1872 
13. Ragan DD, Mates T, Clarke DR (2003) Effect of yttrium and erbium ions on epitaxial phase transformations in alumina. J Am Ceram Soc 86:541-545

14. Maglia F, Gennari S (2008) Energetics of aluminum vacancies and incorporation of foreign trivalent ions in gamma- $\mathrm{Al}_{2} \mathrm{O}_{3}$ : an atomistic simulation study. J Am Ceram Soc 91:283-290

15. Tanabe S, Hanada T (1996) Local structure and $1.5 \mu \mathrm{m}$ quantum efficiency of erbium doped glasses for optical amplifiers. J NonCryst Solids 196:101-105

16. Dimova-Malinovska D, Nichev H, Angelov O, Grigorov V, Kamenova M (2007) Electrical and optical properties of $\mathrm{ZnO}$ thin films prepared by magnetron rf sputtering - influence of $\mathrm{Al}, \mathrm{Er}$ and H. Superlattice Microst 42:123-128

17. Sin JC, Lam SM, Lee KT, Mohamed AR (2013) Fabrication of erbium-doped spherical-like $\mathrm{ZnO}$ hierarchical nanostructures with enhanced visible light-driven photocatalytic activity. Mater Lett 91: $1-4$

18. Ghomri R, Shaikh MN, Ahmed MI, Bououdina M, Ghers M (2016) (Al, Er) co-doped $\mathrm{ZnO}$ nanoparticles for photodegradation of rhodamine blue. Appl Phys A Mater Sci Process 122:895 (9pp)

19. Wang XJ, Lei MK, Yang T, Wang H (2004) Phase structure and photoluminescence $\mathrm{Al}_{2} \mathrm{O}_{3}$ powders prepared by the properties of $\mathrm{Er}^{3+}$-doped sol-gel method. Opt Mater 26:247-252

20. Solehmainen K, Kapulainen M, Heimala P, Polamo K (2004) Erbium-doped waveguides fabricated with atomic layer deposition method. IEEE Phot Technol Lett 16:194-196

21. Wörhoff K, Bradley JDB, Ay F, Geskus D, Blauwendraat TP, Pollnau M (2009) Reliable low-cost fabrication of low-loss $\mathrm{Al}_{2} \mathrm{O}_{3}: \mathrm{Er}^{3+}$ waveguides with $5.4-\mathrm{dB}$ optical gain. IEEE $\mathrm{J}$ Quantum Elect 45:454-461

22. Chryssou CE, Pitt CW (1998) $\mathrm{Er}^{3+}$-doped $\mathrm{Al}_{2} \mathrm{O}_{3}$ thin films by plasma-enhanced chemical vapor deposition (PECVD) exhibiting a 55-nm optical bandwidth. IEEE J. Quantum Elect 34:282-285

23. Mardare CC, Hassel AW (2014) Investigations on bactericidal properties of molybdenum tungsten oxides combinatorial thin film material libraries. ACS Comb Sci 16:631-639

24. Kollender JP, Gallistl B, Mardare AI, Hassel AW (2014) Photoelectrochemical water splitting in a tungsten oxide nickel oxide thin film material library. Electrochim Acta 140:275281
25. Mardare AI, Ludwig A, Savan A, Hassel AW (2014) Properties of anodic oxides grown on a hafnium-tantalum-titanium thin film library. Sci Technol Adv Mater 15:015006

26. Gasiorowski J, Kollender JP, Hingerl K, Sariciftci NS, Mardare AI, Hassel AW (2014) Photoelectrochemical scanning droplet cell microscopy for localized photovoltaic investigations on organic semiconductors. Phys Chem Chem Phys 16:3739-3748

27. Mardare AI, Grill CD, Pötzelberger I, Etzelstorfer T, Stangl J, Hassel AW (2016) Anodic oxide formation on aluminiumterbium alloys. J Solid State Electrochem 20:1673-1681

28. Kollender JP, Voith M, Schneiderbauer S, Mardare AI, Hassel AW (2015) Highly customisable scanning droplet cell microscopes using 3D-printing. J Electroanal Chem 740:53-60

29. Lill KA, Hassel AW (2006) A combined $\mu$-mercury reference electrode/Au counter-electrode system for microelectrochemical applications. J Sol State Electrochem 10:941-946

30. Mardare AI, Hassel AW (2009) Quantitative optical recognition of highly reproducible ultrathin oxide films in microelectrochemical anodization. Rev Sci Instrum 80:046106 (3pp)

31. Mardare AI, Hassel AW (2013) A combinatorial properties study of thin film Al Fe alloys. Phys Status Solidi A 210:1025-1029

32. Mardare AI, Yadav AP, Wieck AD, Stratmann M, Hassel AW (2008) Combinatorial electrochemistry on Al-Fe alloys. Sci Technol Adv Mater 9:035009 (9pp)

33. Pourbaix M (1974) Atlas of electrochemical equilibria in aqueous solution, second. english edition, National Association of Corrosion Engineers, Houston, pp 168-176

34. Chen XB, Cain T, Scully JR, Birbilis N (2014) Technical note: experimental survey of corrosion potentials for rare earth metals $\mathrm{Ce}, \mathrm{Er}, \mathrm{Gd}, \mathrm{La}$, and $\mathrm{Nd}$ as a function of $\mathrm{pH}$ and chloride concentration. Corrosion 70:323-328

35. Lohrengel MM (1993) Thin anodic oxide layers on aluminum and other valve metals - High-field regime. Mater Sci Eng R 11:243294

36. Hsu CS, Mansfeld F (2001) Technical note: concerning the conversion of the constant phase element parameter Y-0 into a capacitance. Corrosion 57:747-748

37. Orazem ME, Shukla P, Membrino MA (2002) Extension of the measurement model approach for deconvolution of underlying distributions for impedance measurements. Electrochim Acta 47: 2027-2034 